\title{
Utilidad de la cápsula endoscópica como método diagnóstico en el estudio de patología de intestino delgado
}

\author{
EDGAR SANHUEZA B. ${ }^{1}$, PATRICIO IBÁÑEZ ${ }^{1}$, RAÚL ARAYA ${ }^{2}$, IRIS DELGADO ${ }^{3 a}$, \\ SOLEDAD QUEZADA $^{1 \mathrm{~b}}$, LILIANA JADUE ${ }^{3}$, CLAUDIO NAVARRETE $^{1}$
}

\section{Experience with capsule endoscopy diagnostic tool for the small intestine}

\begin{abstract}
Background: Wireless capsule endoscopy (CE) is a relatively new method to evaluate the small intestine. Aim: To evaluate the indications of CE in our center and assess whether specific indications are associated with best results during CE studies. Material and Methods: Retrospective analysis of 69 patients aged 9 to 85 years (36 males) subjected to a CE at our institution between April 2004 and October 2007. Results: The most common indications for CE were overt gastrointestinal bleeding in 43.5\% of patients, iron deficiency anemia in $39.1 \%$, suspicion of a small bowel tumor in $4.3 \%$, chronic diarrhea in $4.3 \%$ and abdominal pain in 2.9\%. CE was normal in $23.2 \%$ and was able to find lesions in $76.8 \%$ of the studies. Gastrointestinal bleeding, followed by iron deficiency anemia were the indications associated with the higher rates of positive findings during CE. Conclusions: Gastrointestinal bleeding and iron deficiency anemia were the indications that obtained the best diagnostic yield for CE.

(Rev Med Chile 2010; 138: 303-308).
\end{abstract}

Key words: Capsule endoscopy; Gastrointestinal hemorrhage; Intestine, small.

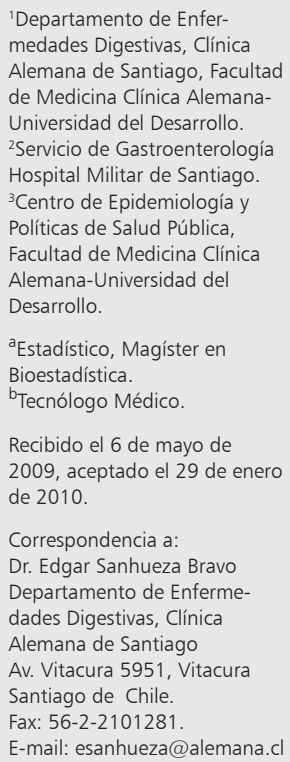

$\mathrm{E}$ 1 desarrollo de la cápsula endoscópica (CE) abre la puerta a la región menos explorada del tubo digestivo, el intestino delgado, que por sus características anatómicas y localización ha sido siempre menos accesible a los estudios endoscópicos ${ }^{1}$. Desde que aparecieron las primeras comunicaciones en voluntarios en el año $2000^{2}$, se abre una nueva etapa en el diagnóstico de enfermedades del intestino delgado, su impacto clínico ha sido reconocido en numerosos trabajos ${ }^{3,4}$, que han demostrado que la $\mathrm{CE}$ es un método efectivo para el diagnóstico de lesiones de la mucosa intestinal ${ }^{5,6}$. Varias publicaciones muestran que la capacidad de detectar lesiones en este segmento del tubo digestivo es superior a las técnicas de imagen tradicionalmente empleadas ${ }^{7-10}$.

Son numerosas las indicaciones de estudio con $\mathrm{CE}$ en patología de intestino delgado, la principal indicación es el estudio de la hemorragia digestiva de origen oculto (HDOO), ya sea en forma evidente o expresada como anemia ferropénica, otras indicaciones menos frecuentes incluyen diarrea crónica y dolor abdominal ${ }^{11}$, en el diagnóstico de enfermedad celíaca y sus complicaciones ${ }^{12-15}$. Según datos aportados por la literatura, la capacidad que tiene este examen de encontrar lesiones en intestino delgado que expliquen con claridad la pérdida de sangre es de aproximadamente $60 \%{ }^{16-}$ ${ }^{18}$. Entre las lesiones más frecuentes que se pueden visualizar, se encuentran lesiones vasculares, erosiones y úlceras. Otro aporte importante es en diagnóstico precoz de la enfermedad inflamatoria intestinal, cuyo aporte también se extiende al control de efectividad en el tratamiento ${ }^{19,20}$.

Este examen fue utilizado por primera vez en seres humanos en $1999^{21}$ y desde agosto del 2001 
fue aprobado por la FDA (Food and Drug Administration de los Estados Unidos de Norteamérica) como método diagnóstico en gastroenterología. Durante los últimos años hemos adquirido experiencia en su uso, sin embargo, a nivel país, su alto costo y escasa disponibilidad determinan ausencia o retardo en la solicitud de este examen. El objetivo principal de nuestro trabajo es evaluar el rendimiento diagnóstico del examen en forma y global y según el motivo para lo cual fue solicitado con lo cual se proporcionará una herramienta útil a los clínicos comprometidos en el estudio de las patologías de intestino delgado.

\section{Material y Método}

Entre abril de 2004 y octubre de 2007, se efectuó un análisis retrospectivo de 71 estudios realizados en 69 pacientes en el Departamento de Enfermedades Digestivas de Clínica Alemana de Santiago. Hubo dos pacientes que requirieron repetir el examen por problemas técnicos, uno por falla de batería y otro por contenido alimentario en tubo digestivo. El examen se efectuó con el equipo "Wireless Capsule Endoscopy" fabricado por Given Imaging, Yoqneam, Israel. El sistema está formado por una cápsula denominada M2A, de $26 \times 11 \mathrm{~mm}$, que el paciente deglute, un aparato registrador externo, una batería y la estación de trabajo, que posteriormente analiza las imágenes. Esta cápsula es impulsada por peristalsis, contiene en su interior un lente y una fuente de luz, y obtiene imágenes de alta resolución a razón de 2 por segundo, durante 8 horas, las cuales son transmitidas mediante radiofrecuencia al registrador adosado al paciente. El procedimiento es ambulatorio y se obtiene un registro topográfico en cuadrantes del abdomen mediante electrodos unidos al cuerpo del paciente. La estación de trabajo permite el análisis de las imágenes ${ }^{21}$ y es capaz de detectar en forma automática cambios de coloración (indicador de rojo) en la mucosa, lo cual facilita el diagnóstico ${ }^{22-24}$. El tiempo estimado de análisis por un operador es variable, dependiendo de la experiencia. El paciente requiere ayuno de $12 \mathrm{~h}$, debe tener la capacidad de deglutir la cápsula, la cual es eliminada en forma natural por las deposiciones entre 12 y 72 horas posteriores a la ingesta. Las contraindicaciones del examen están constituidas por todas aquellas circunstancias que impidan el libre tránsito de la
CE por el tubo digestivo (estenosis, obstrucción intestinal, etc $)^{2}$. Su seguridad en el embarazo aún no ha sido establecida

Los hallazgos fueron definidos según la terminología endoscópica estandarizada ${ }^{26-28}$, y se clasificaron como "positivos" (explican con claridad el cuadro clínico), "sospechosos" (podrían o no explicar el cuadro clínico) y "negativos” (normal) según lo establecido por Penazzio et $\mathrm{al}^{29}$. Todos los pacientes tienen estudio endoscópico alto y bajo sin hallazgos significativos.

Estadística: Para el análisis de cada uno de los 69 estudios se consignó la siguiente información: fecha del estudio; edad y sexo del paciente; motivo por el cual se realizó el examen; diagnóstico por el cual consulta; y el resultado del examen en relación a si este fue alterado (positivo o sospechoso) o negativo (normal). Esta información fue digitada en una base de datos en formato Excel.

Los análisis estadísticos se realizaron con el programa SPSS versión 15.0.

\section{Resultados}

Características del grupo de pacientes: El promedio de edad de los pacientes fue de 55,1 años con una desviación estándar de 19,8 (rango: 9 a 85 años), sin diferencias estadísticamente significativas por sexo. El 52,2\% (36 personas) fueron de sexo masculino.

Motivo del examen: Los dos principales motivos del examen corresponden a HDOO y anemia con $43,5 \%$ y $39,1 \%$ de los pacientes respectivamente. El resto del grupo se divide entre tumores; diarreas; dolor abdominal y otros motivos (Figura 1).

Resultado del examen: De los 69 exámenes analizados, en 53 equivalentes a $76,8 \%$ se obtuvo un resultado alterado (positivo o sospechoso) y en 16 casos, equivalentes a 23,2\%, el resultado del examen fue informado normal (negativo) (Figura 2). Del total de estudios alterados $71,7 \%$ se consideró positivo y $28,3 \%$ sospechoso como causal de patología en intestino delgado (Figura 3). Dos pacientes presentaron complicaciones menores, uno con retención de la cápsula mayor a 48 horas y otro con imposibilidad de tragar.

Diagnósticos encontrados en los exámenes alterados: Considerando el universo de los exámenes alterados (53 casos), en 30,2\% (16 personas) el hallazgo correspondió a angiodisplasia; en otro 
Utilidad de la cápsula endoscópica como método diagnóstico - E. Sanhueza B. et al

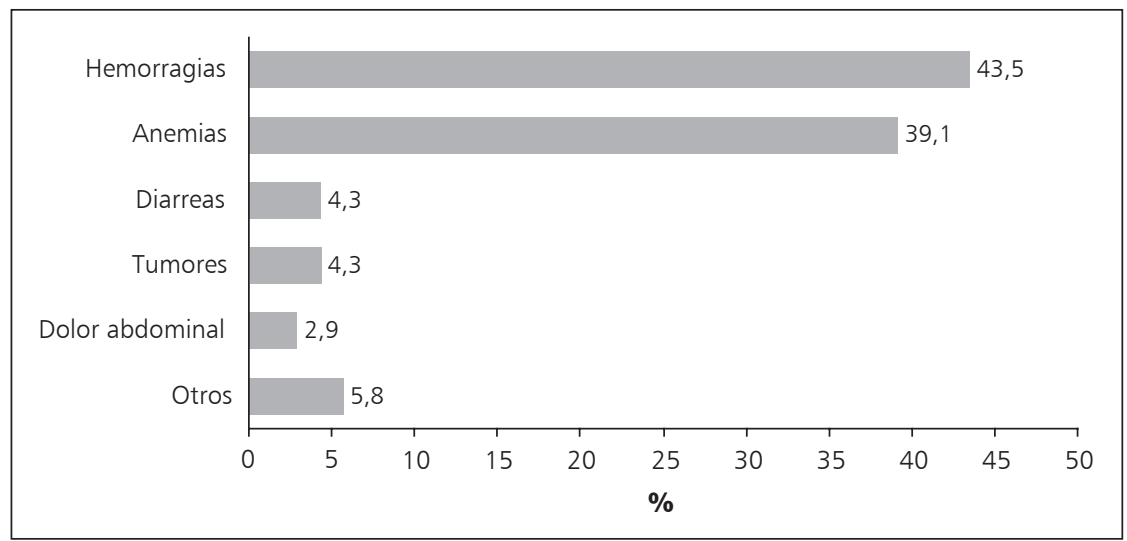

Figura 1. Motivo de realización del examen.

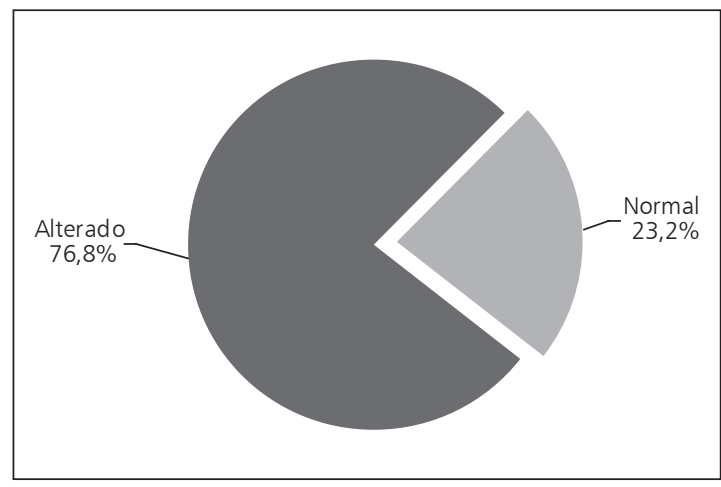

Figura 2. Distribución del resultado del examen según normal o alterado.

26,4\% (14 personas) el resultado del examen correspondió a una lesión protruida (pólipo o tumor). En 10 personas (18,9\%), el hallazgo fue úlcera. Los diagnósticos de fleboectasia, edema y punto rojo, inespecíficos con correlación clínica dudosa, se encontraron en 3 personas. En otros 4 casos el resultado fue no concluyente (Tabla 1).

Resultados según motivo de realización del examen: Este análisis muestra que cuando el motivo de la consulta fue anemia, en $96,3 \%$ de los pacientes el resultado del examen estuvo alterado (sólo en un paciente fue normal). Para el caso de las hemorragias, en tres de cada cuatro personas el resultado fue anormal (76,7\%). En los casos en que el motivo de la consulta fue dolor abdominal, el resultado es bastante inespecífico ya que 50\% resultó ser alterado. En los tres pacientes que consultan por diarrea, el resultado fue normal. (Figura 4).

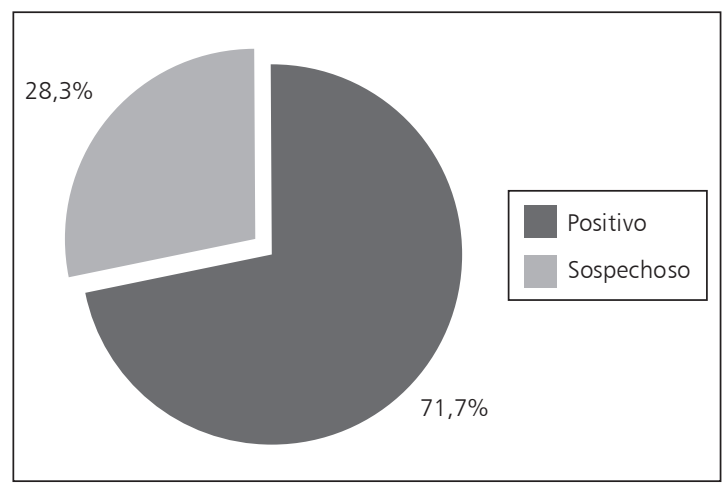

Figura 3. Distribución de resultado alterados según positivo o sospechoso.

Tabla 1. Hallazgos encontrados en exámenes alterados

\begin{tabular}{|lcc|}
\hline & Frecuencia & Porcentaje \\
\hline Angiodisplasia & 16 & 30,2 \\
\hline Lesión protruidas & 14 & 26,4 \\
\hline Úlcera & 10 & 18,9 \\
\hline Punto rojo & 3 & 5,6 \\
Edema & 3 & 5,6 \\
\hline Fleboectasia & 3 & 5,6 \\
\hline No concluyente & 4 & 7,5 \\
\hline Total & 53 & 100 \\
\hline
\end{tabular}


Figura 4. Resultado según motivo por el cual se realizó el examen: Universo: personas con resultado alterado.

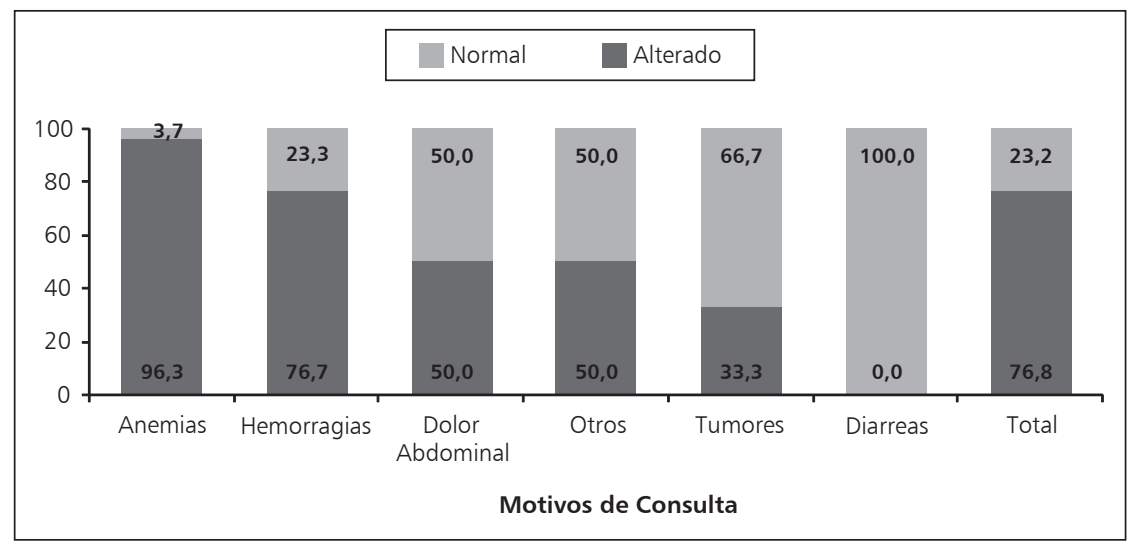

\section{Discusión}

La CE es una modalidad nueva de explorar el intestino delgado, su principal ventaja es la capacidad de visualizar la mucosa con mínimos riesgos para el paciente, y en comparación con el estudio radiológico de intestino delgado, tiene una mayor capacidad de detectar lesiones planas como las angiodisplasias ${ }^{30}$. Las complicaciones descritas para el procedimiento son bajas ${ }^{31}$, la más importante aunque infrecuente es la retención ${ }^{32-35}$. Las desventajas están dadas por su alto costo, la incapacidad de realizar acciones terapéuticas y de establecer con exactitud la relación causa y efecto, al no contar con un patrón de oro en lesiones que por sus características no requieren procedimiento terapéutico invasivo o biopsia (puntos rojos, angiodisplasias no sangrantes, lesiones protruidas inespecíficas de aspecto benigno).

En este escenario es altamente relevante conocer cuál ha sido la utilidad clínica del uso de este método diagnóstico en nuestra práctica, más aun, si durante los últimos años disponemos de métodos diagnósticos orientados a ese segmento del tubo digestivo como la enteroscopia con doble balón y la enteroclisis por tomografía axial computada.

La indicación del examen y la frecuencia de los diagnósticos varían significativamente en diversos estudios $^{36}$. En nuestro trabajo objetivamos que la causa más frecuente por la cual se solicita la CE es por HDOO, similar a lo reportado en la literatu$\mathrm{ra}^{36,37}$, así mismo evidenciamos que la posibilidad de encontrar un hallazgo positivo en este grupo de pacientes es lo suficientemente alta $(73,9 \%)$ como para recomendar su indicación; en contraposición, las técnicas radiológicas convencionales y la arteriografía logran encontrar entre 20 y $51 \%$ de hallazgos positivos ${ }^{38}$. Sigue en frecuencia de solicitud el estudio de anemia crónica de origen ferropénico con o sin presencia de sangre oculta en deposiciones, también con un elevado porcentaje de hallazgos positivos. Para ambas indicaciones los resultados justifican plenamente su uso.

Podemos concluir, entonces, que la utilización del estudio con CE es altamente beneficiosa y similar a lo reportado en la literatura. La capacidad de efectuar diagnóstico global en patología de intestino delgado en nuestro centro fue de $71,7 \%$ en esta serie de pacientes, en que la principal indicación fue el estudio de HDOO y anemia ferropénica.

En nuestra experiencia, la cápsula es un examen útil para el diagnóstico de patología de intestino delgado cuando se indica adecuadamente.

\section{Referencias}

1. Krevsky B. Enteroscopy: exploring the final frontier. Gastroenterology 1991; 100: 838-9.

2. Appleyard M, Glukhovsky A, Swain P. Wireless-capsule diagnostic endoscopy for recurrent small-bowel bleeding. N Engl J Med 2001; 344: 232-3.

3. Liangpunsakul S, Maglinte DDT, Rex DK. Comparison of wireless capsule endoscopy and conventional radiologic methods in the diagnosis of small bowel disease. Gastrointest. Endosc Clin N Am 2004; 14: 43-50.

4. Sturniolo GC, Di Leo V, Vettorato MG, D’Inca R. Clinical 
relevance of small-bowel findings detected by wireless capsule endoscopy. Scand J Gastroenterol 2005; 40: 72533.

5. Appleyard M, Fireman Z, Glukhovsky A, Jacob H, Shreiver R, Kadirkamanathan S, et al. A randomized trial comparing wireless capsule endoscopy with push enteroscopy for the detection of small-bowel lesions. Gastroenterology 2000; 119: 1431-8.

6. Marmo R, Rotondano G, Rondonotti E, de Franchis R, D’Incà R, Vettorato MG, et al. Capsule enteroscopy vs. other diagnostic procedures in diagnosing obscure gastrointestinal bleeding: a cost-effectiveness study. Eur J Gastroenterol Hepatol 2007; 19: 535-42.

7. Voderholzer WA, Ortner M, Rogalla P, Beinhölzl J, Lochs $\mathrm{H}$. Diagnostic yield of wireless capsule enteroscopy in comparison with computed tomography enteroclysis. Endoscopy 2003; 35: 1009-14.

8. Eliakim R, Fischer D, Suissa A, Yassin K, Katz D, Guttman $\mathrm{N}$, et al. Wireless capsule video endoscopy is a superior diagnostic tool in comparison to barium followthrough and computerized tomography in patients with suspected Crohn's disease. Eur J Gastroenterol Hepatol 2003; 15: 363-7.

9. Saperas E, Dot J, Videla S, Álvarez-Castells A, PerezLafuente M, Armengol JR, et al. Capsule endoscopy versus computed tomographic or standard angiography for the diagnosis of obscure gastrointestinal bleeding. Am J Gastroenterol 2007; 102: 731-7.

10. Hara AK, Leighton JA, Sharma VK, Fleischer DE. Small bowel: preliminary comparison of capsule endoscopy with barium study and CT. Radiology 2004; 230: 260-5.

11. May A, Manner H, Schneider M, Ipsen A, Ell C. Prospective multicenter trial of capsule endoscopy in patients with chronic abdominal pain, diarrhea and other signs and symptoms (CEDAP-Plus Study). Endoscopy 2007; 39: 606-12.

12. Hopper AD, Sidhu R, Hurlstone DP, McAlindon ME, Sanders DS. Capsule endoscopy: an alternative to duodenal biopsy for the recognition of villous atrophy in coeliac disease? Dig Liver Dis 2007; 39: 140-5.

13. Rondonotti E, Spada C, Cave D, Pennazio M, Riccioni ME, De Vitis I, et al. Video capsule enteroscopy in the diagnosis of celiac disease: a multicenter study. Am J Gastroenterol 2007; 102: 1624-31.

14. Joyce AM, Burns DL, Marcello PW, Tronic B, Scholz FJ. Capsule endoscopy findings in celiac disease associated enteropathy-type intestinal T-cell lymphoma. Endoscopy 2005; 37: 594-6.

15. Daum S, Wahnschaffe U, Glasenapp R, Borchert M, Ullrich R, Zeitz M, et al. Capsule endoscopy in refractory celiac disease. Endoscopy 2007; 39: 455-8.
16. Lewis BS, Swain P. Capsule endoscopy in the evaluation of patients with suspected small intestinal bleeding: Results of a pilot study. Gastrointest. Endosc 2002; 56: 349-53.

17. Scapa E, Jacob H, Lewkowicz S, Migdal M, Gat D, Gluckhovski A, et al. Initial experience of wireless-capsule endoscopy for evaluating occult gastrointestinal bleeding and suspected small bowel pathology. Am J Gastroenterol 2002; 97: 2776-9.

18. Triester SL, Leighton JA, Leontiadis GI, Fleischer DE, Hara AK, Heigh RI, et al. A meta-analysis of the yield of capsule endoscopy compared to other diagnostic modalities in patients with obscure gastrointestinal bleeding. Am J Gastroenterol 2005; 100: 2407-18.

19. Triester SL, Leighton JA, Leontiadis GI, Gurudu SR, Fleischer DE, Hara AK, et al. A meta-analysis of the yield of capsule endoscopy compared to other diagnostic modalities in patients with non-stricturing small bowel Crohn's disease. Am J Gastroenterol 2006; 101: 954-64.

20. Gay G, Delvaux M, Rey JF. The role of video capsule endoscopy in the diagnosis of digestive diseases: a review of current possibilities. Endoscopy 2004; 36: 913-20.

21. Iddan G, Meron G, Glukhovsky A, Swain P. Wireless capsule endoscopy. Nature 2000; 405 (6785): 417.

22. Liangpunsakul S, Mays L, Rex DK. Performance of Given suspected blood indicator. Am J Gastroenterol 2003; 98: 2676-8.

23. D'Halluin P, Delvaux M, Lapalus M, Sacher-Huvelin S, Ben Soussan E, Heyries L, et al. Does the «Suspected Blood Indicator» improve the detection of bleeding lesions by capsule endoscopy? Gastrointest Endosc 2005; 61: 243-9.

24. Buscaglia JM, Giday SA, Kantsevoy SV, Clarke JO, Magno $\mathrm{P}$, Yong E, et al. Performance characteristics of the suspected blood indicator feature in capsule endoscopy according to indication for study. Clin Gastroenterol Hepatol 2008; 6: 298-301.

25. Ginsberg GG, Barkun AN, Bosco JJ, Isenberg GA, Nguyen CC, Petersen BT, et al. Wireless capsule endoscopy: August 2002. Gastrointest Endosc 2002; 56: 621-4.

26. Delvaux M, Crespi M, Armengol-Miro JR, Hagenmüller F, Teuffel W, Spencer KB, et al. Minimal standard terminology for digestive endoscopy: results of prospective testing and validation in the GASTER project. Endoscopy 2000; 32: 345-55.

27. Delvaux M, Friedman S, Keuchel M, Hagenmüller F, Weinstein M, Cave D, et al. Structured terminology for capsule endoscopy: results of retrospective testing and validation in 766 small-bowel investigations. Endoscopy 2005; 37: 945-50. 
28. Korman LY, Delvaux M, Gay G, Hagenmuller F, Keuchel M, Friedman S, et al. Capsule endoscopy structured terminology (CEST): proposal of a standardized and structured terminology for reporting capsule endoscopy procedures. Endoscopy 2005; 37: 951-9.

29. Pennazio M, Santucci R, Rondonotti E, Abbiati C, Beccari G, Rossini FP, et al. Outcome of patients with obscure gastrointestinal bleeding after capsule endoscopy: report of 100 consecutive cases. Gastroenterology 2004; 126 (3): 643-53.

30. Maglinte DD, Burney BT, Miller RE. Lesions missed on small-bowel follow-through: analysis and recommendations. Radiology 1982; 144: 737-9.

31. Rondonotti E, Herrerias JM, Pennazio M, Caunedo A, Mascarenhas-Saraiva M, de Franchis R. Complications, limitations, and failures of capsule endoscopy: a review of 733 cases. Gastrointest Endosc 2005; 62: 712-716; quiz $752,754$.

32. Li F, Gurudu SR, De Petris G, Sharma VK, Shiff AD, Heigh RI, et al. Retention of the capsule endoscope: a single-center experience of 1000 capsule endoscopy procedures. Gastrointest Endosc 2008; 68: 174-80.

33. Cheifetz AS, Lewis BS. Capsule endoscopy retention: is it a complication? J Clin Gastroenterol 2006; 40: 688-91.

34. Sears DM, Avots-Avotins A, Culp K, Gavin MW. Frequency and clinical outcome of capsule retention during capsule endoscopy for GI bleeding of obscure origin. Gastrointest Endosc 2004; 60: 822-7.

35. Cave D, Legnani P, de Franchis R, Lewis BS. ICCE consensus for capsule retention. Endoscopy 2005; 37: 1065 7.

36. Rastogi A, Schoen RE, Slivka A. Diagnostic yield and clinical outcomes of capsule endoscopy. Gastrointest Endosc 2004; 60: 959-64.

37. Swain P, Adler D, Enns R. Capsule endoscopy in obscure intestinal bleeding. Endoscopy 2005; 37: 655-9.

38. Zuckerman GR, Prakash C, Askin MP, Lewis BS. AGA technical review on the evaluation and management of occult and obscure gastrointestinal bleeding. Gastroenterology 2000; 118: 201-21. 